\title{
Spectral statistics for unitary transfer matrices of binary graphs
}

\author{
Gregor Tanner \\ School of Mathematical Sciences * \\ Division of Theoretical Mechanics \\ University of Nottingham \\ University Park, Nottingham NG7 2RD, UK
}

\begin{abstract}
Quantum graphs have recently been introduced as model systems to study the spectral statistics of linear wave problems with chaotic classical limits. It is proposed here to generalise this approach by considering arbitrary, directed graphs with unitary transfer matrices. An exponentially increasing contribution to the form factor is identified when performing a diagonal summation over periodic orbit degeneracy classes. A special class of graphs, so-called binary graphs, is studied in more detail. For these, the conditions for periodic orbit pairs to be correlated (including correlations due to the unitarity of the transfer matrix) can be given explicitly. Using combinatorial techniques it is possible to perform the summation over correlated periodic orbit pair contributions to the form factor for some low-dimensional cases. Gradual convergence towards random matrix results is observed when increasing the number of vertices of the binary graphs.
\end{abstract}

Submitted to Journal of Physics A; Version: 30th November 1999.

\section{INTRODUCTION}

Universality in spectral statistics has be established numerically and experimentally for a wide range of linear wave problems ranging from quantum systems (Bohigas et al (1984)) to acoustic (Ellegaard et al (1996)) and microwave cavities (Alt et al $(1997,1999)$ ) in two and three dimensions as well as quantum maps (Saraceno and Voros (1994)) and quantum graphs (Kottos and Smilansky $(1997,1998))$, see also Guhr et al (1998) for a recent review. The universality classes are accurately described by random matrix theory (RMT) even though ensemble average is not performed when considering spectra of individual wave problems. This fundamental puzzle is not understood until today and indicates that the RMT-limit is reached under more general conditions than assumed by Wigner, Mehta, Dyson and others (see e.g. Mehta (1991)) in the original derivation of RMT results.

A few basic facts are well established by now: wave systems, whose spectral statistics follow the RMT-result for Gaussian unitary or orthogonal ensemble (GUE or GOE) have in common that

a) time propagation (discrete or continuous) is a linear, unitary transformation;

b) the dynamics of the underlying classical system is chaotic; this implies in particular that the classical Perron-Frobenius operator has an isolated largest eigenvalue equal to one, positive Liapunov exponent and an exponentially increasing number of periodic orbits;

c) there are no systematic periodic orbit length degeneracies other than those enforced by the symmetries of the classical dynamics and the unitarity of the wave propagation.

The last point is kept vague deliberately and refers to systems which fulfill condition (a) and (b) but are known to deviate from RMT due to number theoretical periodic orbit degeneracies; examples are the cat map (Hannay and Berry (1980), Keating (1991 a,b)) and arithmetic billiards of constant negative curvature (Bogomolny et al (1997)). I will come back to this point in the next sections.

\footnotetext{
*e-mail: gregor.tanner@nottingham.ac.uk
} 
A direct consequence of (b) is the so-called Hannay-Ozorio de Almeida (HOdA) sum rule (Hannay and Ozorio de Almeida (1984), Berry (1985)), which enables one to derive universality of the spectral two point correlation function in the long range limit. Considerable progress in understanding the universality of spectral statistics for individual systems beyond the HOdA-sum rule has been made only recently by studying quantum graphs. In a series of papers Smilansky and coworkers demonstrated numerically that quantum graphs indeed obey RMT-statistics (Kottos and Smilansky $(1997,1999))$; they were also able to calculated the full form factor, i.e., the Fourier transform of the spectral two point correlation function, in terms of periodic orbits for a specific set of graphs with $2 \times 2$ unitary transfer matrices (Schanz and Smilansky (1999)) and reproduced Anderson localisation from periodic orbit theory in a similar model (Schanz and Smilansky (1999a)). Deviations from universal statistical behaviour for a special set of graphs - so-called star-graphs could be explained in leading order by Kottos and Smilansky (1999), a systematic way to calculate higher order corrections has been developed by Berkolaiko and Keating (1999).

The main advantage in studying quantum graphs is that one can construct a wide variety of systems with exact periodic orbit trace formulae (in contrast to, for example, semiclassical periodic orbit trace formulae, see Gutzwiller (1990)). Discrete time propagation on a graph corresponds to a unitary transformation in terms of a finite dimensional matrix and periodic orbit lengths are build up by a finite number of rationally independent length segments. The exactness of the trace formula circumvents problems due to, for example, semiclassical errors present in periodic orbit trace formulae for general quantum systems with continuous classical limit. Semiclassical approximations do in general not preserve unitarity of the quantum propagation which leads to exponentially growing error terms in the long time limit (Keating (1994), Tanner (1999)). Periodic orbit length correlations beyond the classical HOdA-sum rules can furthermore be studied in graphs in detail without referring to approximations; such correlations are predicted to exist due to the presence of spectral universality (Argaman et al (1993)).

The quantisation procedure for graphs chosen by Kottos and Smilansky (1997, 1999) implies certain restrictions on the topological structure of the graph. Solving a one-dimensional Schrödinger equation on the connections (or edges) between vertices with various boundary conditions calls for the possibility of backscattering; the underlying graph must therefore be undirected, i.e., the possibility to go from vertex $i$ to vertex $j$ implies that the reversed direction from $j$ to $i$ also exists.

In the following I will broaden the picture by considering unitary matrices in general. I will identify a unitary matrix as a transfer matrix (or 'wave propagator') on a directed graph with exact periodic orbit trace formula. The corresponding classical system is, as for quantum graphs, given by the dynamics on a probabilistic network. Such a construction has a priori, and again like for quantum graphs, no semiclassical limit in the sense that the classical dynamics does not remain the same when increasing the matrix dimension (or the size of the graph). This is, however, not a prerequisite when looking at the conditions (a) - (c); one can indeed easily construct graphs and corresponding unitary transfer matrices which fulfill the conditions above. The main motivation in generalising the concept of quantum graphs lies in the possibility to study a much wider class of graphs including in particular directed graphs. This freedom will be used in section III, IV] to consider a special set of graphs, so-called binary graphs. Unlike for quantum graphs, the unitary transfer matrix of a directed graph can not be written as a function of a wavenumber $k$ in general and does not have a quantum spectrum. Like for quantum maps, one studies instead the statistics of the spectrum of eigen-phases of the unitary matrix.

I will introduce some basic notations for graphs in section II and will define edge and vertex staying rates as well as periodic orbit degeneracy classes. An exponentially increasing contribution to the form factor is identified when performing a diagonal summation over degeneracy classes. I will then focus on balanced, directed (binary) graphs with unitary transfer matrices. The form factor can here be written in terms of a periodic orbit length degeneracy function. This functions will be derived explicitly for binary graphs with up to 6 vertices in section III. Exponentially increasing contributions to the form factor are identified; these contributions alternate in sign and balance each other in a delicate way to lead to an expression for the form factor close to the RMT - result. The periodic orbit form factor for graphs with up to 32 vertices is calculated in section IV by counting the periodic orbit degeneracies directly. Convergence of the periodic orbit expressions towards the RMT - result is observed for graphs with and without time reversal symmetry; this gives rise to the hope that a periodic orbit theory may indeed be able to resolve universality of 
spectral statistics in the limit of large vertex - numbers.

\section{GRAPHS AND UNITARY TRANSFER MATRICES}

\section{A. Introduction and notation}

A directed graph (digraph) $G$ consists of set of vertices $V(G)$ connected by a set of edges $E(G)$. An edge leading from a vertex $i$ to a vertex $j,(i, j \in V(G))$, will be denoted $(i j)$ and the ordering of the pair is important. I will mainly deal with directed graphs here and will omit the specification 'directed' in the following. The order of the graph is given by the number of vertices $N=|V(G)|$, and $M=|E(G)|$ is the number of edges. A graph can be characterised by its $N \times N$ adjacency matrix $\mathbf{A}(G)$ being defined here as

$$
a_{i j}=\left\{\begin{array}{ll}
1 & \text { if } i j \in E(G) \\
0 & \text { otherwise }
\end{array} ;\right.
$$

the vertices $i, j \in V(G)$ may be labeled from 0 to $N-1$ for convenience. A real or complex $N \times N$ matrix $\mathbf{T}(G)$ will be a called a transfer matrix of $G$ if

$$
t_{i j}=0 \Leftrightarrow a_{i j}=0
$$

A real transfer matrix $\mathbf{T}^{c l}(G)$ which preserves probability, i.e.

$$
\sum_{j=0}^{N-1} t_{i j}^{c l}=1 \quad \forall i \in V(G), t_{i j} \in \mathbb{R}
$$

is called a classical transfer matrix in what follows. $\mathbf{T}^{c l}$ is the analogue of the classical transfer or Frobenius-Perron operator for dynamical systems with continuous configuration space and describes the discrete time evolution of an $N$ - dimensional vertex density vector $\boldsymbol{\rho}$ according to

$$
\rho_{j}(n+1)=\sum_{i=0}^{N-1} t_{i j}^{c l} \rho_{i}(n), \quad n \in \mathbb{N} .
$$

A matrix element $t_{i j}^{c l}$ corresponds thus to the transition probability going from vertex $i$ to $j$. The classical transfer matrix has a largest eigenvalue equal to one; the graph is ergodic if there exists a walk or path from $i$ to $j$ for every vertex $i$ and $j$. A graph is 'chaotic' (and thus necessarily ergodic) if the modulus of the second largest eigenvalue is smaller than one. This means, an initial density vector $\boldsymbol{\rho}(0)$ converges exponentially fast towards an equilibrium state $\tilde{\boldsymbol{\rho}}$ which is the eigenvector corresponding to the largest eigenvalue of $\mathbf{T}^{c l}$.

A periodic orbit of period $n$ on a graph is a walk on the graph which repeats after $n$ steps. Each periodic orbit can be labeled in terms of a vertex symbol code $\left(v_{1} v_{2} \ldots v_{n}\right)=\mathbf{v}$ given by the vertices $v_{i} \in V(G)$ visited along the walk with $v_{i} v_{i+1} \in E(G), \forall i=1, n-1$ and $v_{n} v_{1} \in E(G)$. We will denote the set off all periodic orbits of period $n$ as $\mathcal{P} \mathcal{O}_{n}(G)$.

I will in the following focus on unitary transfer matrices $\mathbf{T}$. The 'classical' dynamics corresponding to the 'wave propagation' on the graph described by the unitary matrix $\mathbf{T}$ is then given by the classical transfer matrix $\mathbf{T}^{c l}$ with $t_{i j}^{c l}=\left|t_{i j}\right|^{2}$. The unitarity of $\mathbf{T}$ ensures the probability conservation, Eq. (1), for $\mathbf{T}^{c l}$ and the equilibrium state is the uniform density vector $\tilde{\boldsymbol{\rho}}=(1,1, \ldots 1)$. The complex non-zero matrix elements of $\mathbf{T}$ may be written as $t_{i j}=r_{i j} e^{i L_{i j}}$ and one identifies $L_{i j}$ with the length of an edge $(i j)$ and $r_{i j}^{2}=t_{i j}^{c l}$ is the classical transition probability.

The conditions (a) - (c) in section 1 are fulfilled if the graph is chaotic and the phases $L_{i j}$ are not rationally related apart from conditions enforced due to unitarity. The spectrum of $\mathbf{T}$ and the periodic orbits in the graph are furthermore related by an exact trace formula; the density of states for the eigenphases $\left\{\theta_{i}\right\}_{i=1, N}$ of $\mathbf{T}$ is given as 


$$
d(\theta, N)=\sum_{i=1}^{N} \delta\left(\theta-\theta_{i}\right)=\frac{N}{2 \pi}+\frac{1}{\pi} \operatorname{Re} \sum_{n=1}^{\infty} \operatorname{Tr} \mathbf{T}^{n} e^{-i n \theta}
$$

and the traces $\operatorname{Tr} \mathbf{T}^{n}$ can be written as sum over all periodic orbits of period $n$ in the graph, i.e. $\operatorname{Tr} \mathbf{T}^{n}=\sum_{\mathbf{v} \in \mathcal{P} \mathcal{O}_{n}} A_{\mathbf{v}} e^{i L_{\mathbf{v}}}$. The amplitude $A_{\mathbf{v}}$ is the product over the transition rates $r_{v_{i} v_{i+1}}$ along the path and $L_{\mathbf{v}}$ corresponds to the total length of the periodic orbit.

The spectral measure studied in more detail in this paper is the so-called spectral form factor $K(\tau, N)$; it is the Fourier transformed of the two point correlation function

$$
R_{2}(x, N)=\frac{4 \pi^{2}}{N^{2}}\langle d(\theta) d(\theta+2 \pi x / N)\rangle
$$

and the average $\langle$.$\rangle is taken over the \theta$ - interval $[0,2 \pi]$. The form factor written in terms of periodic orbits has the form (see e.g. Tanner (1999))

$$
K(\tau, N)=\frac{1}{N}\left\langle\left|\operatorname{Tr} \mathbf{T}^{n}\right|^{2}\right\rangle_{\Delta \tau}=\frac{1}{N}\left\langle\sum_{\mathbf{v}, \mathbf{v}^{\prime} \in \mathcal{P} \mathcal{O}_{n}} A_{\mathbf{v}} A_{\mathbf{v}^{\prime}} e^{i\left(L_{\mathbf{v}}-L_{\mathbf{v}^{\prime}}\right)}\right\rangle_{\Delta \tau}
$$

with $\tau$ taking on the discrete values $\tau=n / N$ and further averaging over small intervals $\Delta \tau$ is performed. Most periodic orbits of the graph will be uncorrelated and the corresponding periodic orbit pair contributions will vanish after performing the $\tau$ - average. There are, however, correlations in the periodic orbit length spectrum which lead to systematic deviations from a zero mean; the most obvious one is between orbits which are related by cyclic permutation of the vertex code v. The sum over those pairs of orbits leads to the HOdA - sum rule and describes the linearised behaviour of $K(\tau)$ for $\tau \rightarrow 0$ (Berry (1985)). One can immediately identify another class of exactly degenerated orbits on graphs; this is the set of periodic orbits which passes through each edge the same number of times but not necessarily in the same order. After defining the so-called edge staying rates $q_{i j}$ of $\mathbf{v}$ as the number of times a give orbit $\mathbf{v}$ visits a certain edge (ij), i.e.

$$
q_{i j}(\mathbf{v})=\sum_{l=1}^{n} \delta_{i, v_{l}} \delta_{j, v_{l+1}} \quad(i j) \in E(G) \quad \mathbf{v} \in \mathcal{P O}_{n}
$$

one can write the length $L_{\mathbf{v}}$ and the amplitude $A_{\mathbf{v}}$ of an orbit $\mathbf{v}$ on a graph as

$$
L_{\mathbf{v}}=\sum_{i j \in E(G)} q_{i j}(\mathbf{v}) L_{i j}, \quad A_{\mathbf{v}}=\prod_{i j \in E(G)} r_{i j}^{q_{i j}(\mathbf{v})} .
$$

Periodic orbits whose symbol string gives rise to the same edge staying rate vector $\mathbf{q}=$ $\left(\left\{q_{i j}\right\}_{i j \in E(G)}\right)$ coincide in length $L_{\mathbf{v}}$ and amplitude $A_{\mathbf{v}}$; these orbits will be called topologically degenerate. The set of all topologically degenerated orbits will be called a degeneracy class (Berkolaiko and Keating (1999)). The number of orbits in a given degeneracy class represented by the $M$ dimensional edge staying rate vector $\mathbf{q}$ (with $M$, the number of edges of the graph) will be denoted the (periodic orbit length) degeneracy function $P_{n}(\mathbf{q} ; G)$, i.e.

$$
P_{n}(\mathbf{q} ; G)=\left|\left\{\mathbf{v} \in \mathcal{P} \mathcal{O}_{n} \mid q_{i j}(\mathbf{v})=q_{i j}, \forall i j \in E(G)\right\}\right| .
$$

The orbits related by cyclic permutation of the symbol code are obviously in the same degeneracy class.

The traces of $\mathbf{T}$ which enter the density of states (2) can thus be rewritten as

$$
\operatorname{Tr} \mathbf{T}^{n}=\sum_{\mathbf{q} \in \mathbb{K}_{n}(G)} P_{n}(\mathbf{q}) A_{\mathbf{q}} e^{i L_{\mathbf{q}}}
$$

and $\mathbb{K}_{n}(G) \subset \mathbb{N}_{0}^{M}$ represents the subset of the M-dimensional integer lattice $\mathbb{N}_{0}^{M}$ containing all the possible edge staying rate vectors $\mathbf{q}$ which correspond to periodic orbits of period $n$ of the graph $G$. Determining the lattice $\mathbb{K}_{n}(G)$ and thus the possible degeneracy classes as well as the degeneracy 
function is the main problem when studying periodic orbit length correlations on graphs. I will come back to this point in the next section.

The form factor (3) can now be written as double sum over the edge rate vectors $\mathbf{q}$

$$
K(n, N)=\frac{1}{N}\left\langle\sum_{\mathbf{q}, \mathbf{q}^{\prime} \in \mathbb{K}_{n}(G)} A_{\mathbf{q}} A_{\mathbf{q}^{\prime}} P_{n}(\mathbf{q}) P_{n}\left(\mathbf{q}^{\prime}\right) e^{i\left(L_{\mathbf{q}}-L_{\mathbf{q}^{\prime}}\right)}\right\rangle_{\Delta \tau} .
$$

A new type of diagonal contribution emerges when considering periodic orbit pairs sharing a common $\mathbf{q}$ - vector. The total contribution of topologically degenerate periodic orbit pairs, which obviously includes the original diagonal contributions in the HOdA - sum rule, is

$$
K_{\text {top }}(n, N)=\frac{1}{N} \sum_{\mathbf{q} \in \mathbb{K}_{n}(G)} A_{\mathbf{q}}^{2} P_{n}^{2}(\mathbf{q}) \sim e^{\alpha_{t} n}
$$

and $\alpha_{t}>0$ in general; (the rate $\alpha_{t}$ can be calculated using large deviation techniques (Dembo and Zeitouni (1993)), strict upper and lower bounds are $0 \leq \alpha_{t} \leq h_{t}$, and $h_{t}$ is the topological entropy for the graph). All the contributions to $K_{t o p}$ are positive which coincides with a result obtained by Whitney et al (1999) using diagrammatic techniques for periodic orbit formulae. The diagonal approximation $K_{t o p} \sim \frac{n}{N}$ following from the HOdA - sum rule is valid only for small $\tau=\frac{n}{N}$ when cyclic permutation is the main source of degeneracies. (This is in general the case for those $n$ values for which the majority of orbits visits a given edge at most once).

Unitarity of the underlying $\mathbf{T}$ matrix implies the asymptotic result $\lim _{\tau \rightarrow \infty} K(\tau, N)=1$; the exponentially increasing topological contributions $K_{t o p}$ must therefore be counterbalanced by additional correlations in the periodic orbit length spectrum. We will show that these kind of correlations originate from the unitarity of the $\mathbf{T}$ matrix and that the cancelation mechanism is extremely sensitive leaving little space for approximate or asymptotic treatments.

All what has been said so far is true for arbitrary unitary matrices, and thus especially for transfer matrices of quantum graphs and also for general quantum maps. In order to study the phenomena of periodic orbit correlations due to unitarity more closely, I will now focus on a special class of chaotic graphs with uniform transition probabilities for which all relevant periodic orbit correlations can be given explicitly.

\section{B. Binary graphs and periodic orbit correlations}

One of the simplest, non-trivial class of graphs are balanced, directed binary graphs $B_{N}$; these are connected graphs with $N$ vertices ( $N$ even) for which each vertex has exactly two incoming and two outgoing edges. The adjacency matrix $\mathbf{A}_{N}$ of a binary graph can be written in the form

$$
a_{i j}=\left\{\begin{array}{ll}
\delta_{2 i, j}+\delta_{2 i+1, j} & \text { for } 0 \leq i<\frac{N}{2} \\
\delta_{2 i-N, j}+\delta_{2 i+1-N, j} & \text { for } \frac{N}{2} \leq i<N
\end{array} \quad i=0, \ldots, N-1\right.
$$

and the number of edges of $B_{N}$ is $M=2 N$. Some examples together with their adjacency matrices are shown in Figs. 2, 1, and 5. It will sometimes be useful to switch from a vertex code to an edge code. A suitable choice is to assign each edge $i j$ corresponding to a non-zero matrix element of the adjacency matrix (9) an edge code

$$
i_{e}=2 i+j \bmod 2, \quad i_{e}=0,1 \ldots, 2 N-1
$$

The edge code is given for the examples Figs. 2, 4, and 5 .

Transfer matrices of binary graphs have been studied in connection with combinatorial problems for binary sequences (Stanley (1999)), as well as the semiclassical quantisation of the Anisotropic Kepler Problem using binary symbolic dynamics (Gutzwiller (1988), Tanner and Wintgen (1995)) and have been discussed in the context of general quantum maps (Bogomolny (1992)). Saraceno (1999) recently proposed a quantisation scheme for the baker map which also leads to quantum maps of the form (9).

Binary graphs with adjacency matrices (9) are connected, i.e., each vertex can be reached from every other vertex, here after at least $\left[\log _{2} N\right]+1$ steps. The topological entropy $h_{t}=\log 2$ 
independent of the order of the graph. The subset of binary graphs of order $N=2^{k}, k \in \mathbb{N}$, the so-called de Bruijn - graphs (Stanley (1999)), deserves special attention; the dynamics on these graphs can directly be related to the set of all binary sequences and there exists a one-to-one relation between finite binary symbol strings $\left(a_{1}, a_{2}, \ldots a_{n}\right), a_{i} \in\{0,1\}$ of length $n$ and the periodic orbits of the graph i.e.

$$
\left(a_{1}, a_{2}, \ldots a_{n}\right) \leftrightarrow\left(v_{1}, v_{2}, \ldots v_{n}\right) \quad a_{i} \in\{0,1\}, v_{i} \in\left\{0,2^{k}-1\right\}
$$

with

$$
v_{i}=\sum_{j=1}^{k} a_{i+j-1} 2^{k-j} \quad \text { and } a_{i+n}=a_{i}
$$

for graphs of order $N=2^{k}$. The number of orbits of period $n$ on these graphs is exactly $2^{n}$.

I will consider unitary transfer matrices of binary graphs next. The unitarity conditions for a transfer matrix $\mathbf{T}_{N}$ of a binary graph with adjacency matrix (9) can be stated simply in terms of the unitarity conditions for the set of $N / 2$ different $2 \times 2$ matrices $\mathbf{u}_{i}$ with

$$
\mathbf{u}_{i}=\left(\begin{array}{ll}
t_{i, 2 i} & t_{i, 2 i+1} \\
t_{i+\frac{N}{2}, 2 i} & t_{i+\frac{N}{2}, 2 i+1}
\end{array}\right) \quad i=1,2, \ldots, N / 2 .
$$

Focusing on unitary binary transfer matrices with uniform local spreading, i.e., setting $\left|t_{i j}\right|=1 / \sqrt{2}$ for all non-zero matrix elements of $\mathbf{T}_{N}$, the unitarity condition can be written as a pure phase correlation. One obtains the following relation between the lengths of edges

$$
\left(L_{i, 2 i}+L_{i+\frac{N}{2}, 2 i+1}\right)-\left(L_{i, 2 i+1}+L_{i+\frac{N}{2}, 2 i}\right) \bmod 2 \pi=\pi \quad i=0,2, \ldots, N / 2-1,
$$

the corresponding local network is shown in Fig. 1. The unitary condition (12) will be shown to be responsible for the periodic orbit correlations relevant to balance out the exponentially increasing topological contributions to the form factor $K(\tau, N)$. Its simplicity makes it possible to turn the problem of finding periodic orbit length correlations into a combinatorial problem of finding all exact periodic orbit degeneracies (up to phase differences being a multiple of $\pi$ ), which can be done in principle.

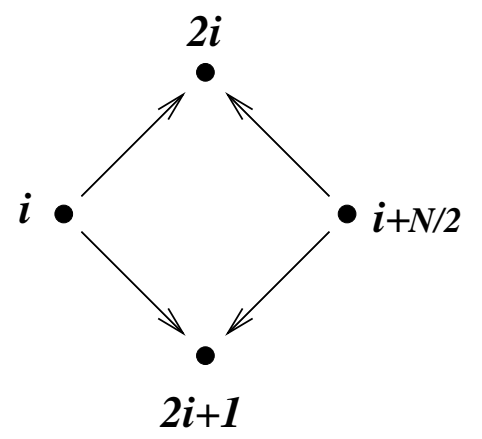

FIG. 1. Local network of correlated edge lengths, see Eq. (12); opposite edges form a pair, the two pairs have a combined length difference of $\pi$.

The dynamics described by the classical transfer matrix with transition probabilities $t_{i j}^{c l}=1 / 2$ for all possible transitions $i j$ in the binary graph is maximally mixing for the geometry (9), i.e. $h_{t}=K=\log 2$ and $K$ is the Kolmogorov entropy for graphs (Schuster (1989)); the conditions (a) (c) in section I are thus satisfied as long as there are no systematic edge length correlations present except from those introduced through Eq. (12). One can furthermore show that the generalised diagonal contribution (8) increases exponentially with a rate $\alpha_{t}=h_{t}=\log 2$ independent of the order of the binary graph. 
Periodic orbit correlations beyond topological degeneracies can be expressed in terms of edge and vertex staying rates. The vertex staying rates $\tilde{q}_{i}(\mathbf{v})$ of an orbit $\mathbf{v}$ are defined analogue to (4) as the number of times a periodic orbit visits a vertex $i$, i.e.

$$
\tilde{q}_{i}(\mathbf{v})=\sum_{l=1, n} \delta_{i, v_{l}} \quad i \in V(G) .
$$

Vertex and edge staying rates are connected by conservation laws (or shift invariance properties (Dembo and Zeitouni (1993))) of the form

$$
\begin{aligned}
q_{i, 2 i}+q_{i, 2 i+1}=q_{\left[\frac{i}{2}\right], i}+q_{\left[\frac{i}{2}\right]+\frac{N}{2}, i}=\tilde{q}_{i} & \forall i=0, \ldots, \frac{N}{2}-1 \\
\underbrace{q_{i, 2 i-\frac{N}{2}}+q_{i, 2 i-\frac{N}{2}+1}}=\underbrace{q_{\left[\frac{i}{2}\right], i}+q_{\left[\frac{i}{2}\right]+\frac{N}{2}, i}}=\tilde{q}_{i} & \forall i=\frac{N}{2}, \ldots, N-1
\end{aligned}
$$

incoming edges outgoing edges

and [.] denotes the integer part. A direct consequence of (12) and (14) is the following condition for periodic orbit correlations:

All periodic orbits having the same vertex staying rates $\tilde{\mathbf{q}}=\left(\tilde{q}_{0}, \ldots, \tilde{q}_{N-1}\right)$ differ in length exactly by a multiple of $\pi$.

This can be shown by noting that for two orbits $\mathbf{v}, \mathbf{v}^{\prime} \in \mathcal{P} \mathcal{O}_{n}\left(B_{N}\right)$ with $\Delta \tilde{\mathbf{q}}=\tilde{\mathbf{q}}(\mathbf{v})-\tilde{\mathbf{q}}\left(\mathbf{v}^{\prime}\right)=\mathbf{0}$, one obtains

$$
\Delta q_{i, 2 i}+\Delta q_{i, 2 i+1}=0, \quad \Delta q_{i, 2 i}+\Delta q_{i+\frac{N}{2}, 2 i}=0, \quad \Delta q_{i+\frac{N}{2}, 2 i+1}+\Delta q_{i+\frac{N}{2}, 2 i}=0,
$$

see also Fig. 1. One therefore has

$$
\Delta q_{i, 2 i}=\Delta q_{i+\frac{N}{2}, 2 i+1}=-\Delta q_{i, 2 i+1}=-\Delta q_{i+\frac{N}{2}, 2 i}
$$

which together with (12) yields

$$
\Delta L=L_{\mathbf{v}}-L_{\mathbf{v}^{\prime}}=\pi \sum_{i=0}^{N / 2-1} \Delta q_{i, 2 i}=\pi d_{\mathbf{v}, \mathbf{v}^{\prime}} .
$$

The corresponding contribution of the periodic orbit pair to the form factor (3) is then $(-1)^{d_{\mathbf{v}}, \mathbf{v}^{\prime}} 2^{-n}$. Note that the amplitudes $A_{\mathbf{v}}$ equal $2^{-n / 2}$ for all orbits of period $n$.

The from factor can thus be written as a sum over weighted correlations of the degeneracy function (5), i.e.

$$
\begin{aligned}
K(n, N) & =\frac{1}{N} \frac{1}{2^{n}} \sum_{\tilde{\mathbf{q}} \in \tilde{\mathbb{K}}_{n}\left(B_{N}\right)}\left(\sum_{\mathbf{q}} \sum_{\mathbf{q}^{\prime}}(-1)^{\sum_{i} \Delta q_{i, 2 i}} P_{n}(\mathbf{q}) P_{n}\left(\mathbf{q}^{\prime}\right)\right) \\
& =\frac{1}{N} \frac{1}{2^{n}} \sum_{\tilde{\mathbf{q}} \in \tilde{\mathbb{K}}_{n}\left(B_{N}\right)}\left(\sum_{\mathbf{q}}(-1)^{\left[\sum_{i} q_{i, 2 i}\right]} P_{n}(\mathbf{q})\right)^{2}
\end{aligned}
$$

and [.] denotes the integer part. The sum is taken over the $N$ dimensional integer lattice $\tilde{\mathbb{K}}_{n}\left(B_{N}\right)$ of possible vertex staying rate vectors $\tilde{\mathbf{q}}$ corresponding to periodic orbits of period $n$ of a binary graph $B_{N}$; the vectors $\mathbf{q}, \mathbf{q}^{\prime}$ correspond here to the $N / 2$ components $\left(q_{i, 2 i}, i=0, N / 2-1\right)$ of the total edge staying rate vector only. The contributions of periodic orbit pairs which are not correlated by having length differences being a multiple of $\pi$ are assumed to be Gaussian distributed with zero mean. We will neglect these random background contributions from now on and concentrate on the contributions from correlated periodic orbit pairs only. 
Before turning to the problem of calculating degeneracy functions, a few remarks on the edge staying rates. The components of the edge staying rate vector $\mathbf{q}$ are related to each other by the shift invariance properties (14). These are $N$ conditions which can be shown to lead to $N-1$ independent equations for the $2 N$ rates $q_{i j}$; together with the restriction

$$
\sum_{i=0}^{N-1} \tilde{q}_{i}=n
$$

for orbits of period $n$, one can write the edge staying rates in terms of $N$ independent quantities, which effectively allows to half the dimension of $\mathbb{K}_{n}\left(B_{N}\right)$. The length degeneracy functions $P_{n}$ depends thus on $N$ independent variables only.

There are further restrictions on the independent components of q. Apart from the obvious condition $q_{i j} \geq 0 \forall i j \in E\left(B_{N}\right)$, one must also ensure that the sum over the $N$ independent components of $\mathbf{q}$ does not exceed $n$ and that the staying rates do correspond to a connected, closed path on the graph. An example for an edge staying rate vector $\mathbf{q}$ violating the last restriction is $\mathbf{q}=$ $\left(q_{00}, 0, \ldots, 0, q_{N-1, N-1}\right)$ with $q_{00} \neq 0$ and $q_{N-1, N-1} \neq 0$ which corresponds to two disconnected periodic orbits. I will come back to the problem of determining the lattice $\mathbb{K}_{n}$ in more detail in the next section.

\section{PERIODIC ORBIT LENGTH DEGENERACY FUNCTIONS - ANALYTIC RESULTS}

The periodic orbit length correlations in binary graphs with constant transition amplitudes can be completely described in terms of the degeneracy function (5). The problem of calculating the form factor is thus converted to a combinatorial problem of finding the number of closed (connected) paths on a graph which visit each edge the same number of times. This problem can be treated explicitly for low-dimensional graphs; results for binary graphs up to order 6 will be presented here.

\section{A. Binary graphs of order $N=2$}

The case $N=2$ has already been treated by Schanz and Smilansky (1999) in somewhat different circumstances. 17 We will re-derive some of the results in order to introduce the basic notations and concepts which will be useful when considering binary graphs for $N>2$. Some new asymptotic results for the two-dimensional case will also be presented here.

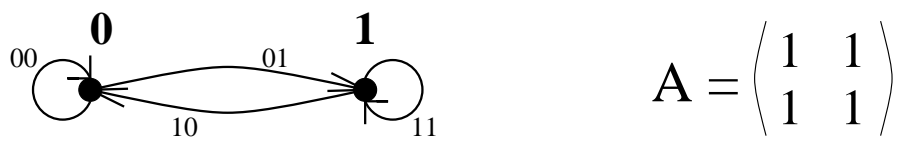

FIG. 2. Binary graph of order 2 together with its adjacency matrix A.

A binary graph of order 2 is shown in Fig. 2. The shift invariance property, Eq. (14), implies the following conditions for the edge staying rate vector $\mathbf{q}=\left(q_{00}, q_{01}, q_{10}, q_{11}\right)$, i.e.

$$
\begin{aligned}
& \tilde{q}_{0}=q_{00}+q_{01}=q_{10}+q_{00}, \\
& \tilde{q}_{1}=q_{11}+q_{10}=q_{01}+q_{11}
\end{aligned}
$$

and $\tilde{q}_{0}, \tilde{q}_{1}$ represent the vertex staying rates. After choosing $q_{00}$ and $q_{11}$ as independent variables and together with the condition (17), one obtains

\footnotetext{
${ }^{1}$ Schanz and Smilansky (1999) analysed unitary $2 \times 2$ matrices in connection with simple quantum (star-) graphs. The unitary transfer matrices considered have the extra constraint $L_{01}=L_{10}$. It can, however, be shown that this conditions does not lead to additional periodic orbit length correlations, see also Sec. IV.
} 


$$
q_{01}=q_{10}=\frac{1}{2}\left(n-q_{00}-q_{11}\right), \quad \tilde{q}_{0}=\frac{1}{2}\left(n+q_{00}-q_{11}\right), \quad \tilde{q}_{1}=\frac{1}{2}\left(n-q_{00}+q_{11}\right),
$$

for orbits of period $n$.

The periodic orbit length degeneracy function $P_{n}\left(q_{00}, q_{11}\right)$ can be derived by starting with the special case $q_{00}=q_{11}=0$. One immediately obtains $P_{n}(0,0)=2$ for $n$ even; the two periodic orbits correspond to the $\frac{n}{2}$-th repetition of the primitive periodic orbits 01 and 10 of period 2 . It is advantageous to switch to an edge symbol code, i.e., to identify

$$
00 \rightarrow 0_{e} ; \quad 01 \rightarrow 1_{e} \quad 10 \rightarrow 2_{e} ; \quad 11 \rightarrow 3_{e}
$$

see also Eq. 10 and Fig. 2. The two orbits 01 and 10 can then be written as

$$
\underbrace{1_{e} 2_{e} 1_{e} 2_{e} \ldots 1_{e} 2_{e}}_{n} \text {, and } \underbrace{2_{e} 1_{e} 2_{e} 1_{e} \ldots 2_{e} 1_{e}}_{n} \text {. }
$$

The symbol $0_{e}$ can only occur after the symbol $2_{e}$ and it can be repeated. A periodic orbit of period $n+q_{00}$ can thus be obtained by inserting $q_{00}$ symbols $0_{e}$ in between the $2_{e} 1_{2}$ blocks in the periodic orbit sequences (20). Symbols $0_{e}$ can be placed at $\frac{n}{2}+1$ positions for the first orbit in (20) and $\frac{n}{2}$ positions for the second orbit. Similar arguments apply for inserting $q_{11}$ symbols $3_{e}$ into the sequences (20). Using standard combinatorial formulae to find the number of combinations to distribute $q_{00}$ items among $\frac{n}{2}+1$ or $\frac{n}{2}$ boxes with repetitions, one obtains

$$
P_{n+q_{00}+q_{11}}\left(q_{00}, q_{11}\right)=\left(\begin{array}{c}
\frac{n}{2}+q_{00} \\
q_{00}
\end{array}\right)\left(\begin{array}{c}
\frac{n}{2}+q_{11}-1 \\
q_{11}
\end{array}\right)+\left(\begin{array}{c}
\frac{n}{2}+q_{00}-1 \\
q_{00}
\end{array}\right)\left(\begin{array}{c}
\frac{n}{2}+q_{11} \\
q_{11}
\end{array}\right) .
$$

After rescaling $\left(n+q_{00}+q_{11}\right)$ to $n$ and using the relations (19) one may write the degeneracy function as

$$
P_{n}\left(q_{00}, q_{11}\right)=\frac{n}{q_{01}}\left(\begin{array}{c}
\tilde{q}_{0}-1 \\
q_{00}
\end{array}\right)\left(\begin{array}{c}
\tilde{q}_{1}-1 \\
q_{11}
\end{array}\right)
$$

The possible integer values for $q_{00}$ and $q_{11}$ have to obey certain restrictions which follow directly from (19), i.e.

$$
q_{00}+q_{11}<n \text { and }\left(n-q_{00}-q_{11}\right) \bmod 2=0 .
$$

The degeneracy function (21) approaches a Gaussian distribution in the limit $n \rightarrow \infty$; its form can be derived with the help of large deviation techniques (Dembo and Zeitouni (1993)), i.e., one obtains

$$
\begin{aligned}
& P_{n}\left(q_{00}, q_{11}\right) \sim \frac{4}{\pi n} 2^{n} e^{-n\left(4 x^{2}+y^{2}\right)} \\
& \text { with } \quad x=\frac{1}{n \sqrt{2}}\left(q_{00}+q_{11}-\frac{n}{2}\right), \quad y=\frac{1}{n \sqrt{2}}\left(q_{00}-q_{11}\right) .
\end{aligned}
$$

The asymptotic result (23) is too crude to be useful in a calculation of the form factor directly; it does provide, however, some insight into the asymptotic behaviour of the various contributions entering the form factor. Especially the contributions of topologically degenerate periodic orbit pairs, see Eq. (8), can be estimated to be

$$
K_{\text {top }}(n) \sim \frac{1}{2^{n+1}} \iint d q_{00} d q_{11} P_{n}^{2}\left(q_{00}, q_{11}\right)=\frac{2^{n}}{\pi n},
$$

and one obtains $\alpha_{t}=\log 2$ for the growth rate of the diagonal contributions (8). Periodic orbit pairs being degenerate up to a phase difference $m \pi$ enter the form factor asymptotically as

$$
K_{m}(n) \sim \frac{(-1)^{m}}{2^{n+1}} \iint d q_{00} d q_{11} P_{n}\left(q_{00}, q_{11}\right) P_{n}\left(q_{00}+m, q_{11}+m\right)=(-1)^{m} \frac{2^{n}}{\pi n} e^{-4 \frac{m^{2}}{n}} .
$$


The form factor thus consists of an increasing number of exponentially growing terms which differ in sign (see also Fig. 3). Only a very delicate balance between these terms ensures the cancelations necessary to lead to the asymptotic behaviour $\lim _{n \rightarrow \infty} K(n)=1$. The approximations above are indeed not sufficient to preserve the asymptotic limit and give exponentially growing terms for large $n$; similar arguments might hold for the breakdown of semiclassical approximations to quantum form factors, see e.g. Tanner (1999). Note also, that the diagonal terms relevant for the HOdA sum rule do not play a prominent role in the discussion above; they give a linear contribution to $K_{t o p}$ which is already sub-dominant for moderate $n$ values.

The periodic orbit pair contributions to the form factor can be computed explicitly by summing the exact length degeneracy function (21) over the possible edge staying rates obtained from the conditions (22). It may be written in compact form in the following way (Schanz and Smilansky (1999))

$$
K(n)=\frac{1}{2^{n+1}}\left[2+\sum_{\tilde{q}_{0}=1}^{n-1}\left(\sum_{q_{01}=1}^{\left.\frac{n}{2}-\left|\frac{n}{2}-\tilde{q}_{0}\right|\right)}(-1)^{q_{01}} P_{n}\left(q_{00}, q_{11}\right)\right)^{2}\right]=1+\frac{(-1)^{n+l}}{2^{2 l+1}}\left(\begin{array}{c}
2 l \\
l
\end{array}\right),
$$

with $l=[n / 2]$ and $\tilde{q}_{0}, q_{01}$ can be expressed in terms of $q_{00}, q_{11}$ using (19). It is a remarkable fact that the sum can be determined explicitly, a result derived by Schanz and Smilansky (1999) using quantum graph techniques. The form factor, Eq. (24), is displayed in Fig. 3 together with the asymptotic results. $K(n)$ approaches 1 in the large $n$ limit, but is different from the RMTresult for $2 \times 2$ matrices. The periodic structure can be seen to coincide with the start of a new family of degenerate orbits and is thus a remnant of non-perfect cancelation of the various $K_{m}(n)$ contributions. Convergence of the correlated periodic orbit pair contributions to the RMT - result is observed when increasing the order $N$ of the binary graph as will be shown in the next sections.

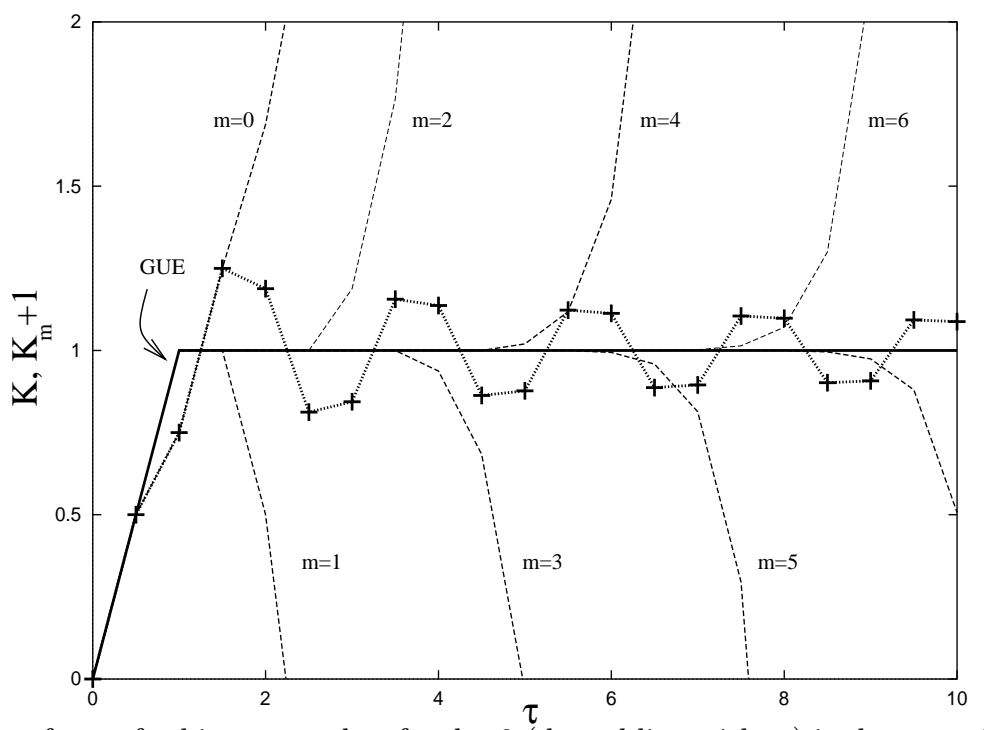

FIG. 3. The form factor for binary graphs of order 2 (dotted line with + ) is shown as function of $\tau=\frac{n}{2}$; the partial sums $K_{m}$ contribute with alternating signs starting at $\tau=m+1$; (the dashed lines correspond to $K_{m}+1$ for $m>0$ ). The GUE - form factor is also displayed for comparison.

\section{B. Binary graphs of order $N=4$ and $N=6$}

The edge and vertex staying rates of periodic orbits of a binary graph of order $N=4$, see Fig. 4 , can be written in terms of 4 independent variables. A possible choice for the edge staying rates is $q_{00}, q_{12}, q_{21}$ and $q_{33}$. The other edge and vertex rates can be computed by using Eqs. (14), explicit formulae are given in appendix A. 


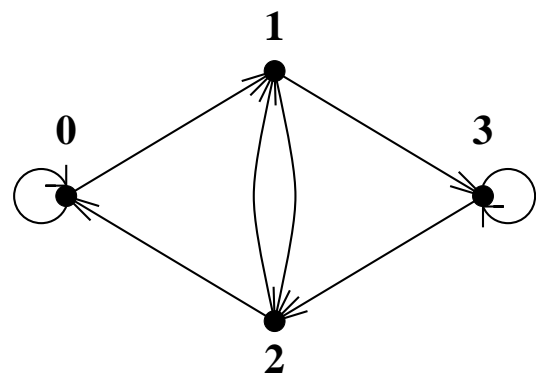

$$
A=\left(\begin{array}{llll}
1 & 1 & 0 & 0 \\
0 & 0 & 1 & 1 \\
1 & 1 & 0 & 0 \\
0 & 0 & 1 & 1
\end{array}\right)
$$

FIG. 4. Binary graph of order 4 together with its adjacency matrix A. The subscript $e$ denotes the edge symbol code.

The periodic orbit length degeneracy function can be obtained by arguments similar to the one described in the last section. The discussion is somewhat technical and is referred to appendix A. The final result is

$$
P_{n}\left(q_{00}, q_{12}, q_{21}, q_{33}\right)=\frac{n}{\tilde{q}_{1}}\left(\begin{array}{c}
\tilde{q}_{1} \\
q_{01}
\end{array}\right)\left(\begin{array}{c}
\tilde{q}_{2} \\
q_{21}
\end{array}\right)\left(\begin{array}{c}
\tilde{q}_{0}-1 \\
q_{00}
\end{array}\right)\left(\begin{array}{c}
\tilde{q}_{3}-1 \\
q_{33}
\end{array}\right),
$$

and $\tilde{q}_{i}$ denotes again the vertex staying rates. The possible entries on the 4-dimensional integer $\mathbf{q}$ lattice can be stated by conditions similar to those in Eq. (22). Periodic orbits which differ in length by a multiple of $\pi$ have the same vertex staying rates but may differ in the variables

$$
s_{0}=q_{00}+q_{21}, \quad s_{1}=q_{12}+q_{33} .
$$

The length difference for orbits with identical vertex rates is given by $\Delta L=\frac{1}{2}\left(\Delta s_{0}+\Delta s_{1}\right) \pi$, see Eq. (15). The form factor can be written in terms of degenerate periodic orbit pairs only and one obtains

$$
K(n)=\frac{1}{4} \frac{1}{2^{n}}\left(2+\sum_{\tilde{q}_{0}+\tilde{q}_{1}=1}^{n-1} \sum_{\tilde{q}_{1}=1}^{\left.\frac{n}{2}-\left|\frac{n}{2}-\tilde{q}_{0}-\tilde{q}_{1}\right|\right)}\left(\sum_{s_{0}=\left|q_{00}-q_{21}\right|, s_{1}=\left|q_{12}-q_{33}\right|}^{s_{0}+s_{1}<n}(-1)^{\left[\frac{s_{0}+s_{1}}{2}\right]} P_{n}(\mathbf{q})\right)^{2}\right) .
$$

The form factor $K(\tau)$ with $\tau=n / 4$ obtained from Eq. (27) is shown in Fig. 6. It oscillates periodically with decreasing amplitude about the RMT - result similar to the behaviour observed in the case $N=2$, see Fig. 3. A closed expression for the sum similar to Eq. (27) could not be found.

The sums in (27) are already quite cumbersome and the number of summation variables increases with the order $N$. The number and complexity of the restrictions for the $\mathbf{q}-$ lattice $\mathbb{K}_{n}\left(B_{N}\right)$ increases accordingly. The case $N=6$ can, however, still be treated along the ideas developed above; it will be presented here as a last example for obtaining the form factor by summing over the periodic orbit length degeneracy function.

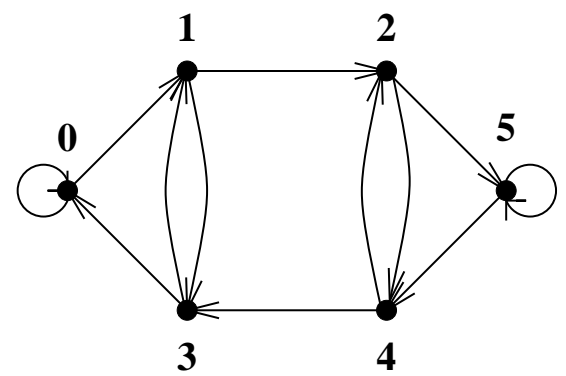

$$
A=\left|\begin{array}{llllll}
1 & 1 & 0 & 0 & 0 & 0 \\
0 & 0 & 1 & 1 & 0 & 0 \\
0 & 0 & 0 & 0 & 1 & 1 \\
1 & 1 & 0 & 0 & 0 & 0 \\
0 & 0 & 1 & 1 & 0 & 0 \\
0 & 0 & 0 & 0 & 1 & 1
\end{array}\right|
$$

FIG. 5. Binary graph of order 6 together with its adjacency matrix A. The subscript $e$ denotes the edge symbol code.

The binary graph of order $N=6$ is shown in Fig. 5 . A possible choice for the independent edge staying rates is $q_{00}, q_{13}, q_{24}, q_{31}, q_{42}$, and $q_{55}$. The derivation of the degeneracy function can again be found in appendix A, the final result is 


$$
P_{n}(\mathbf{q})=\frac{n q_{12}}{\tilde{q}_{2} \tilde{q}_{3}}\left(\begin{array}{c}
\tilde{q}_{1} \\
q_{31}
\end{array}\right)\left(\begin{array}{c}
\tilde{q}_{2} \\
q_{42}
\end{array}\right)\left(\begin{array}{c}
\tilde{q}_{3} \\
q_{13}
\end{array}\right)\left(\begin{array}{c}
\tilde{q}_{4} \\
q_{24}
\end{array}\right)\left(\begin{array}{c}
\tilde{q}_{0}-1 \\
q_{00}
\end{array}\right)\left(\begin{array}{c}
\tilde{q}_{5}-1 \\
q_{55}
\end{array}\right)
$$

The vertex rates $\tilde{q}_{i}$ and the edge rate $q_{12}$ entering (28) can be expressed in terms of the independent variables $\mathbf{q}=\left(q_{00}, q_{13}, q_{24}, q_{31}, q_{42}, q_{55}\right)$, see appendix A. The summation over the six dimensional lattice $\mathbb{K}_{n}\left(B_{6}\right)$ of possible $\mathbf{q}$ vectors can be stated in terms of the vertex staying rates and the variables

$$
s_{0}=q_{00}+q_{31}, \quad s_{1}=q_{12}+q_{43}, \quad s_{2}=q_{24}+q_{55} .
$$

The expression for the form factor as sum over degenerate periodic orbit pairs is thus

$$
K(n)=\frac{1}{6} \frac{1}{2^{n}} \sum_{\tilde{q}_{0}=1}^{n} \sum_{\tilde{q}_{1}=0}^{\left[\left(n-\tilde{q}_{0}\right) / 2\right]} \sum_{\tilde{q}_{2}=0}^{\left[\left(n-\tilde{q}_{0}-2 \tilde{q}_{1}\right) / 2\right]}\left(\sum_{s_{0}, s_{1}, s_{2}}^{\left(s_{0}+s_{1}+s_{2}\right)<n}(-1)^{\left[\frac{s_{0}+s_{1}+s_{2}}{2}\right]} P_{n}(\mathbf{q})\right)^{2}
$$

and the inner sum runs over all possible $s_{i}, i=0,1,2$ values. The form factor $K(\tau)$ after summing Eq. (29) is displayed in Fig. 6 with $\tau=n / 6$. The sums, Eqs. (27) and (29), follow the RMT result more closely than in the $N=2$ case, see Fig. 3. The linear behaviour for $\tau<1$ starts to emerge and convergence to the asymptotic result $K \rightarrow 1$ is observed in the large $\tau=n / N$ limit.

Larger matrices have to be considered in order to test convergence of degenerate periodic orbit pair contributions towards the RMT form factor for all $\tau$. Determining the degeneracy function and the lattice conditions $\mathbb{K}_{n}\left(B_{N}\right)$ becomes increasingly difficult for graphs of order $N>6$. In the next section, I will therefore present results obtained from counting all correlated periodic orbit pair contributions directly.

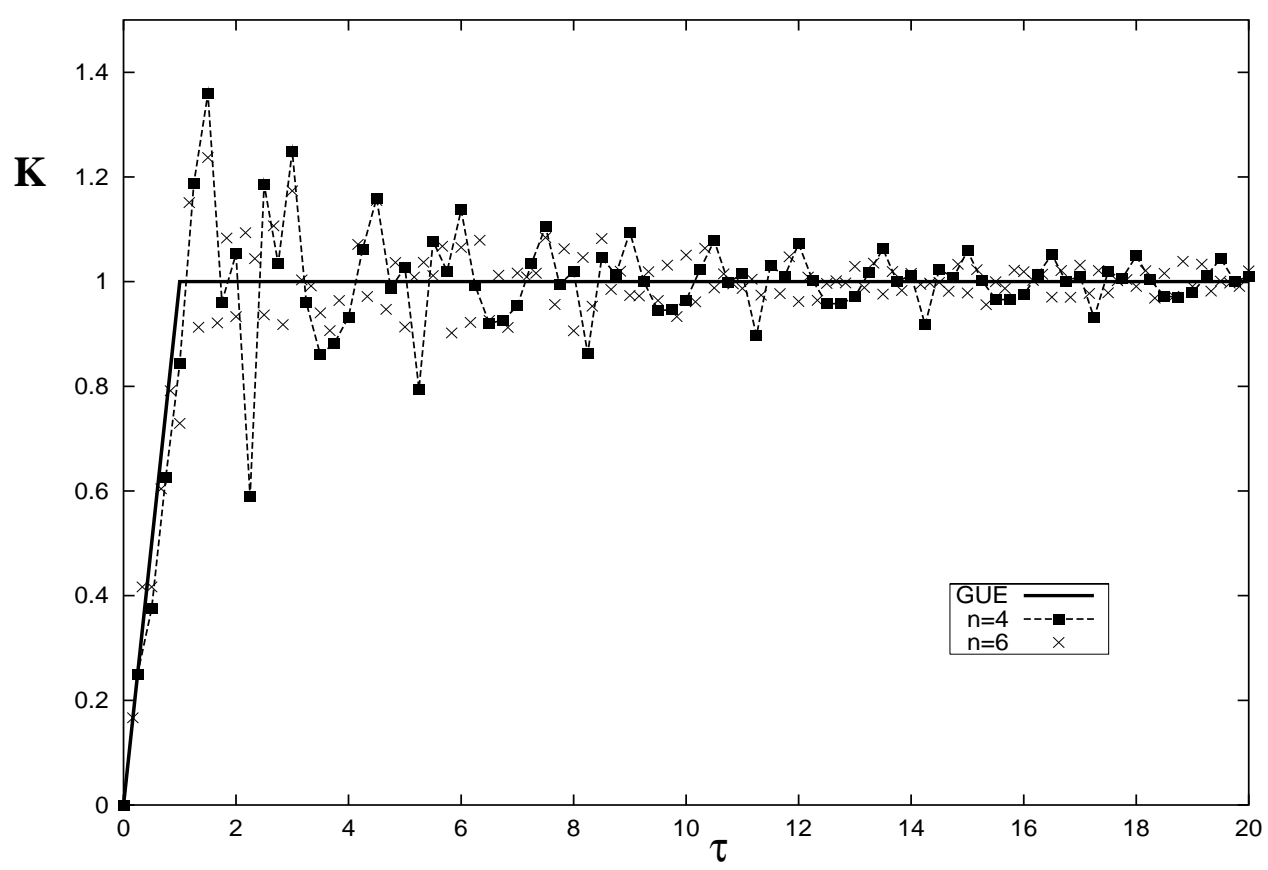

FIG. 6. Periodic orbit pair contributions to the form factor for binary graphs of order $N=4$ and $N=6$.

\section{PERIODIC ORBIT PAIR CONTRIBUTIONS TO THE FROM FACTOR FOR DE BRUIJN GRAPHS OF ORDER $N \geq 8$.}

The periodic orbit pair contributions to the form factor can be calculated directly by determining the set of periodic orbits of given period $n$ and calculating periodic orbit degeneracies with the help of edge and vertex staying rates and the condition (15). The task of finding the set of periodic 
orbits is especially simple for de Bruijn graphs, i.e. for binary graphs of order $N=2^{r}$, due to the one-to-one relation between periodic orbits and finite binary symbol strings, see section II B.

Counting the periodic orbit pair degeneracies explicitly does, however, seriously limit the range of periods over which periodic orbit correlations can be considered. Due to the exponential increase in the number of orbits only values up to $n \approx 26$ could be reached. This in turn sets an upper bound on the $\tau=n / N$ values for which the form factor can be studied.

\section{GUE - results:}

Results for $N=8,16$ and 32 and no further symmetry present are shown in Fig. 7. One observes a convergence of the periodic orbit pair contributions to the GUE-result; the kink at $\tau=1$ is resolved for binary graphs of order $N=16$; the periodic orbit results follows the linear behaviour for $\tau<1$ even closer for $N=32$. It was not possible to extend the results for $n=32$ to the critical time $\tau=1$ due to the restrictions on the available $n$ values. The small $\tau$ behaviour is dominated by the exponentially increasing topological contributions, see Fig. 7. The so-called diagonal contributions due to cyclic permutations of periodic orbit codes are important only in the small $\tau$ regime, i.e. $\tau \sim \log _{2}(N) / N$, before vertex exchange degeneracies set in.

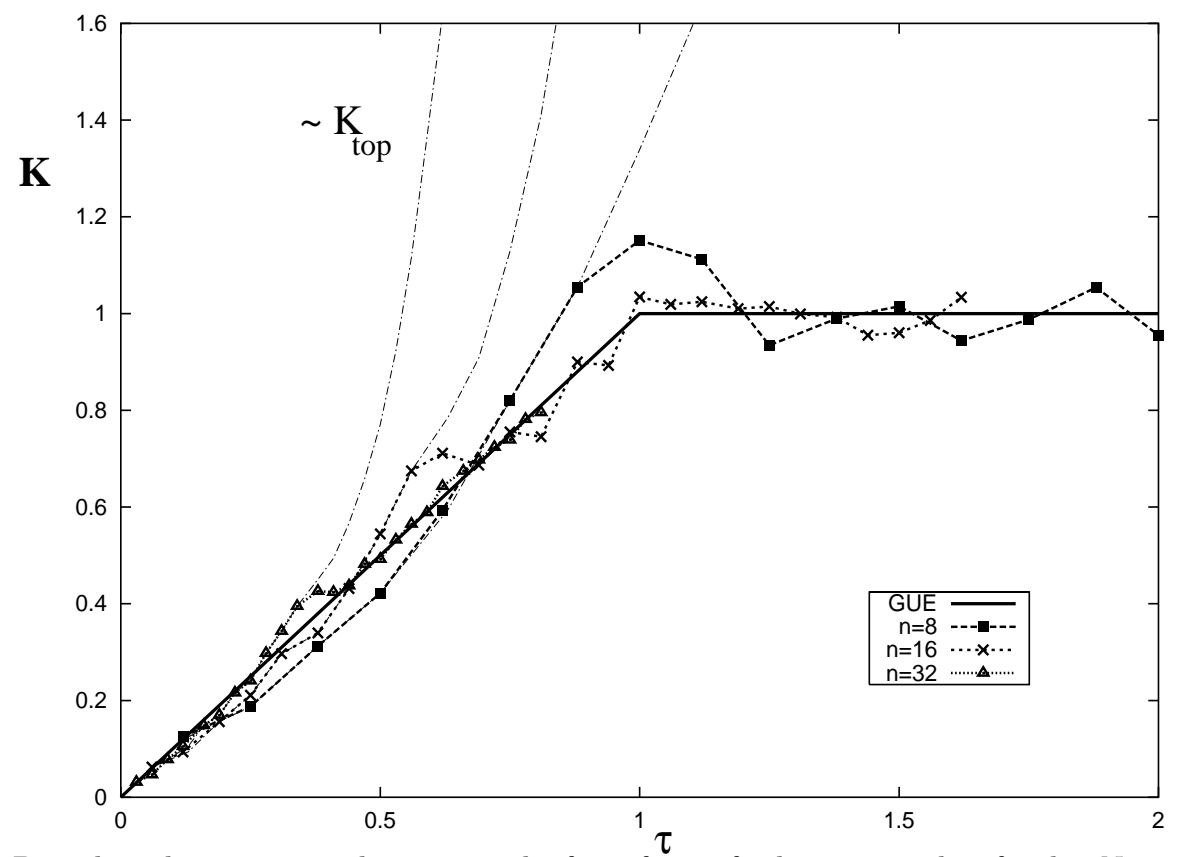

FIG. 7. Periodic orbit pair contributions to the form factor for binary graphs of order $N=8,16$ and 32 . The small $\tau$ behaviour is dominated by exponentially increasing topological contributions (dashed lines).

GOE - results:

So far only unitary transfer matrices without symmetries have been considered. Symmetries in the dynamics impose additional correlations on periodic orbit length spectra and do have an effect on the spectral statistics. Time reversal symmetry is of special importance as it occurs frequently in physical systems; correlations due to time reversal symmetry are in addition non-trivial leading to a form factor which is not piecewise linear as in the GUE case; only the linear behaviour for $K(\tau)$ in the limits $\tau \rightarrow 0$ and $\tau \rightarrow \infty$ is understood in terms of semiclassical arguments (Berry (1985)).

It is a priori not clear how to establish time reversal symmetry for the dynamics on an arbitrary directed graph. Time reversal symmetry can, however, be constructed for de Bruijn graphs of order $N=2^{k}$ using the underlying binary symbolic dynamics and the edge code, Eq. (10). The edge code can be written in terms of a binary string of length $k+1$ such that $i_{e}=\sum_{l=1}^{k+1} a_{l}\left(i_{e}\right) 2^{k+1-l}$, and $\mathbf{a}\left(i_{e}\right)=\left(a_{1}, \ldots a_{k+1}\right)$ is a binary string of length $k+1$ with $a_{l} \in 0,1$. Time reversal symmetry can be established by imposing

$$
L_{i_{e}}=L_{i_{e}^{\prime}}, r_{i_{e}}=r_{i_{e}^{\prime}} \quad \text { if } \quad \mathbf{a}\left(i_{e}\right)=\overline{\mathbf{a}}\left(i_{e}^{\prime}\right)
$$


for the edge lengths and transition rates and $\overline{\mathbf{a}}$ denotes the code $\mathbf{a}$ written backwards, i.e. $i_{e}^{\prime}=$ $\sum_{l=1}^{k+1} a_{l}\left(i_{e}\right) 2^{l-1}$. The condition, Eq. (30), and the unitarity condition, Eq. (12), are assumed to be the only sources of correlations in the periodic length spectrum.

Time reversal symmetry does not effect graphs of the order $N \leq 8, N=2^{k}$. This is due to the fact that the edge staying rates for a given edge and its time reversed partner are related by conservation laws (14) such that there are no further degeneracies for these low dimensional cases. One finds for $N=2$, for example, that $q_{01}=q_{10}$ and a periodic orbit and its time reversed partner are always in the same degenaracy class even if the condition (30) is not imposed.

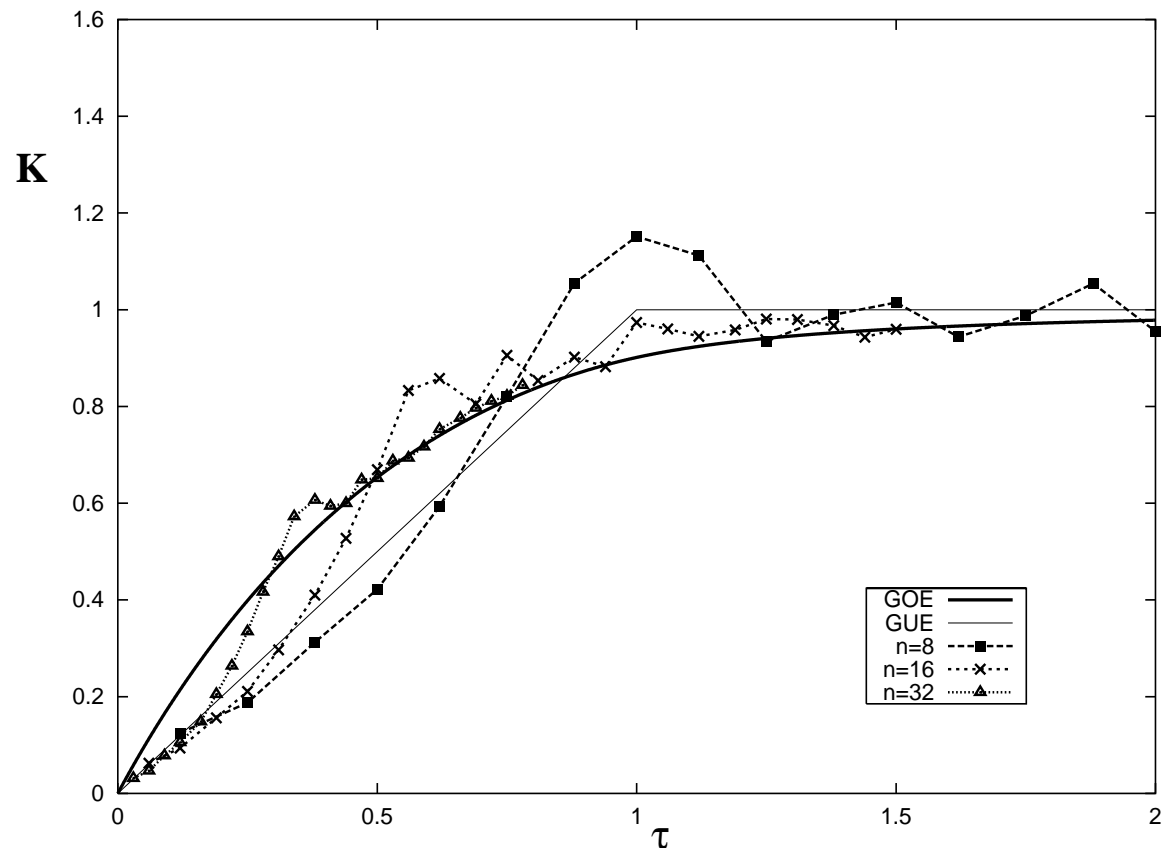

FIG. 8. Periodic orbit pair contributions to the form factor for binary graphs of order $N=8, N=16$ and $N=32$ and time reversal symmetry.

Results for graphs with time reversal symmetry are shown in Fig. 8; the case $N=8$ is indeed identical to the non-time reversal symmetric result in Fig. 7 . The results for $N=16$ and 32 are, however, different from those in Fig 7; the periodic orbit pair contributions approach the GOE - result and not the GUE form factor with increasing $N$. The condition (30) does therefore introduces new correlations among periodic orbits for $N>8$ which are beyond the additional topological degeneracy between an orbit and its time reversed partner giving rise to a factor 2 in the HOdA - diagonal approximation. Note also the exponentially increasing components for small $\tau$ due to topological degeneracies similar to those in Fig. 8.

\section{CONCLUSIONS}

Degeneracies in the length spectrum of periodic orbits of generic directed graphs have been studied. Transition rates and edge lengths in the graph are identified with the amplitudes and phases of matrix elements of the complex transition matrices. General concepts like edge and vertex staying rates as well as the periodic orbit length degeneracy function have been introduced. The form factor can be written in terms of the degeneracy function revealing an exponentially increasing 'diagonal contribution' due to topologically degenerated orbits. Topological degeneracies

\footnotetext{
${ }^{2}$ The unitary $2 \times 2$ matrices studied by Schanz and Smilansky (1999) do thus correspond to time reversal symmetric (binary) graphs.
} 
exist independently of the actual choice of length segments on the graph (defined through the transition matrix) and are a purely 'classical' effect depending only on the topology of the graph. Further correlations amongst orbits are introduced when considering unitary transfer matrices.

These correlations have been studied for a particular simple class of graphs, so-called binary graphs with constant transition amplitudes. The correlations can be given explicitly in terms of edge and vertex staying rates. One finds in particular that periodic orbits which have the same vertex staying rates differ in length by exactly a multiple of $\pi$. Finding the periodic orbit degeneracy function turns into a combinatorial problem which has been solved for binary graphs with up to 6 vertices.

The form factor can be shown to consist of exponentially increasing contributions which balance in a very delicate way to give $\lim _{\tau \rightarrow \infty} K(\tau)=1$. The periodic orbit sums also reveal convergence towards the RMT - result for intermediate $\tau$ - values when increasing the order of the graph, both for time reversal and non-time reversal symmetric binary graphs. Binary graphs may thus turn out to be an ideal model systems to study the connection between periodic orbit formulae and random matrix theory. Eigen spectra of binary graphs follow generic random matrix statistics in the large $N$ limit and periodic orbit correlations are known explicitly.

\section{Acknowledgments}

Parts of the work has been carried out at BRIMS, Hewlett-Packard Laboratories, Bristol; I would like to thank Jeremy Gunawardena for the hospitality experienced throughout my stays, Neil O'Connell for stimulating discussions and Gregory Berkolaiko for comments on the manuscript.

References

[1] Alt H, Dembowski C, Graef H-D, Hofferbert R, Rehfeld H, Richter A, Schuhmann R and Weiland T 1997 Phys. Rev. Lett. 79, 1026

[2] Alt H, Dembowski C, Graef H-D, Hofferbert R, Rehfeld H, Richter A, Schmit C 1999 Phys. Rev. E 60, 2851

[3] Argaman N, Dittes F-M, Doron E, Keating J P, Kitaev A Yu, Sieber M and Smilansky U 1993 Phys. Rev. Lett. 71 4326-4329

[4] Berkolaiko G and Keating J P 1999 J. Phys. A 32 7827-7814

[5] Berry M V 1985 Proc. R. Soc. A 400 229-51

[6] Bogomolny E 1992 Nonlinearity 5 805-66

[7] Bogomolny E, Georgeot B, Giannoni M J and Schmit C 1997, Phys. Rep. 291 220-340

[8] Bohigas O, Giannoni M J and Schmit C 1984 Phys. Rev. Lett. 52, 1-5

[9] Dembo A and Zeitouni D 1993 Large Deviations Techniques (Jones and Bartlett, Boston)

[10] Ellegaard C, Guhr T, Lindemann K, Nygaard J and Oxborrow M, 1996 Phys. Rev. Lett. 774918.

[11] Guhr T, Müller-Groeling A and Weidenmüller H A 1998 Phys. Rep. 299 189-428.

[12] Gutzwiller M C 1988 J Phys. Chem. 92 3154-3163

[13] Gutzwiller M C 1990 Chaos in Classical and Quantum Mechanics (Springer, New York).

[14] Hannay J H and Berry M V 1980 Physica D 1 267-290

[15] Hannay J H and Ozorio de Almeida A M 1984 J. Phys. A 17 3420-9

[16] Keating J P 1991 Nonlinearity 4 277-307

[17] - 1991 Nonlinearity 4 309-41

[18] Keating J P 1994 J. Phys. A 27 6605-6615

[19] Kottos T and Smilansky U 1997 Phys. Rev. Lett. 79 4794-4797

[20] - 1999 Ann. of Phys. 274 76-124

[21] Mehta M L 1991 Random Matrices (2nd ed., Academic Press, New York)

[22] Saraceno M and Voros A 1994 Physica D 79 206-268

[23] Saraceno M 1999 private communication

[24] Schanz H and Smilansky U 1999 to be published in the Proceedings of the Australian Summer School on Quantum Chaos and Mesoscopics, Canberra, Australia, January 1999 
25] Schanz H and Smilansky U 1999a Periodic-Orbit Theory of Anderson Localization on Graphs chaodyn/9909023

[26] Schuster H G 1989 Deterministic Chaos (1st reprint of 2nd ed, VCH, Weinheim)

[27] Stanley R P 1999 Enumerative Combinatorics Vol 2 (Cambridge University Press)

[28] Tanner G and Wintgen D 1995 Chaos, Solitons \& Fractals 5 1325-1336

[29] Tanner G 1999 J. Phys. A 32 5071-5085

[30] Whitney R S, Lerner I V and Smith R A 1999 Can the trace formula describe weak localisation? cond-mat/9902328.

\section{APPENDIX A: PERIODIC ORBIT LENGTH DEGENERACY FUNCTION FOR BINARY GRAPHS: EXACT RESULTS}

The expressions for periodic orbit length degeneracy functions for binary graphs of order $N=4$ and $N=6$, Eqs. (25) and (28), will be derived here.

$\underline{\text { The case } N=4}$ :

As for binary graphs of order $N=2$ discussed in section III A, it is useful to switch to an edge symbol code, see Eq. (10); adopting the vertex symbol code of Fig. 4, one defines the edges as

$$
\begin{aligned}
& 00 \rightarrow 0_{e} ; \quad 01 \rightarrow 1_{e} \quad 12 \rightarrow 2_{e} ; \quad 13 \rightarrow 3_{e} ; \\
& 20 \rightarrow 4_{e} ; \quad 21 \rightarrow 5_{e} \quad 32 \rightarrow 6_{e} ; \quad 33 \rightarrow 7_{e} .
\end{aligned}
$$

A suitable set of independent edge staying rates is $q_{0_{e}}, q_{2_{e}}, q_{5_{e}}, q_{7_{e}}$ and I will drop the subscript $e$ as long as there is no confusion with the vertex code. The remaining edge and vertex staying rates for periodic orbits of period $n$ can be written in terms of the edge staying rates above with the help of Eqs. (14), i.e., one obtains

$$
\begin{aligned}
& q_{1}=q_{4}=\frac{1}{4}\left(n-q_{0}+q_{2}-3 q_{5}-q_{7}\right) \\
& q_{3}=q_{6}=\frac{1}{4}\left(n-q_{0}-3 q_{2}+q_{5}-q_{7}\right)
\end{aligned}
$$

for the edge rates and

$$
\begin{aligned}
\tilde{q}_{0} & =\frac{1}{4}\left(n+3 q_{0}+q_{2}-3 q_{5}-q_{7}\right) \\
\tilde{q}_{1}=\tilde{q}_{2} & =\frac{1}{4}\left(n-q_{0}+q_{2}+q_{5}-q_{7}\right) \\
\tilde{q}_{3} & =\frac{1}{4}\left(n-q_{0}-3 q_{2}+q_{5}+3 q_{7}\right)
\end{aligned}
$$

for the vertex staying rates $\tilde{q}_{i}$ and the index $i$ denotes the vertex code, here.

The periodic orbit length degeneracy function $P_{n}\left(q_{0}, q_{2}, q_{5}, q_{7}\right)$ can be computed by starting from

$$
P_{n}\left(0, \frac{n}{2}, \frac{n}{2}, 0\right)=2 \quad \text { for } n \text { even ; }
$$

the set of edge staying rates above corresponds to the orbits of length $n$ with edge symbol code

$$
2525 \ldots 25 \text { and } 525 \ldots 252 \text {. }
$$

One proceeds by noting that an edge symbol ' 2 ' in the sequences (A3) can be replaced by the sequence ' 36 ' to give an orbit of length $n+1$. Similarly one may substitute a symbol ' 5 ' by the sequence '4 1'. Replacing $m$ symbols '2' and $k$ symbols ' 5 ', $m, k \leq \frac{n}{2}$, one obtains

$$
P_{n+m+k}\left(0, \frac{n}{2}-m, \frac{n}{2}-k, 0\right)=2\left(\begin{array}{c}
\frac{n}{2} \\
m
\end{array}\right)\left(\begin{array}{c}
\frac{n}{2} \\
k
\end{array}\right)+\left(\begin{array}{c}
\frac{n}{2}-1 \\
m-1
\end{array}\right)\left(\begin{array}{c}
\frac{n}{2} \\
k
\end{array}\right)+\left(\begin{array}{c}
\frac{n}{2} \\
m
\end{array}\right)\left(\begin{array}{c}
\frac{n}{2}-1 \\
k-1
\end{array}\right),
$$


and the last two terms in the sum come from orbits which start with a symbol ' 6 ' or a symbol ' 1 ', respectively. After replacing $n+m+k$ by the new periodic orbit length $n^{\prime}$, i.e., $n=n^{\prime}-m-k$ and writing $\tilde{q}_{1}=\frac{1}{4}\left(n^{\prime}+q_{2}+q_{5}\right)$ with $q_{2}=\frac{1}{2}\left(n^{\prime}-3 m-k\right), q_{5}=\frac{1}{2}\left(n^{\prime}-m-3 k\right)$ one obtains

$$
P_{n^{\prime}}\left(0, q_{2}, q_{5}, 0\right)=2\left(\begin{array}{c}
\tilde{q}_{1} \\
m
\end{array}\right)\left(\begin{array}{c}
\tilde{q}_{1} \\
k
\end{array}\right)+\left(\begin{array}{c}
\tilde{q}_{1}-1 \\
m-1
\end{array}\right)\left(\begin{array}{c}
\tilde{q}_{1} \\
k
\end{array}\right)+\left(\begin{array}{c}
\tilde{q}_{1} \\
m
\end{array}\right)\left(\begin{array}{c}
\tilde{q}_{1}-1 \\
k-1
\end{array}\right) .
$$

Next, one notes that an edge symbol ' 0 ' or ' 7 ' can be inserted between any symbol ' 4 ' and ' 1 ' or ' 3 ' and ' 6 ', respectively, to obtain a periodic orbit of length $n^{\prime}+1$. Inserting $q_{0}$ symbols ' 0 ' and $q_{7}$ symbols ' 7 ' into $k$ sequences ' 41 ' and $m$ sequences ' 36 ' (with repetition) leads to

$$
\begin{aligned}
P_{n^{\prime}+q_{0}+q_{7}}\left(q_{0}, q_{2}, q_{5}, q_{7}\right) & =2\left(\begin{array}{c}
\tilde{q}_{1} \\
m
\end{array}\right)\left(\begin{array}{c}
\tilde{q}_{1} \\
k
\end{array}\right)\left(\begin{array}{c}
m+q_{7}-1 \\
q_{7}
\end{array}\right)\left(\begin{array}{c}
k+q_{0}-1 \\
q_{0}
\end{array}\right) \\
& +\left(\begin{array}{c}
\tilde{q}_{1}-1 \\
m-1
\end{array}\right)\left(\begin{array}{c}
\tilde{q}_{1} \\
k
\end{array}\right)\left(\begin{array}{c}
m+q_{7} \\
q_{7}
\end{array}\right)\left(\begin{array}{c}
k+q_{0}-1 \\
q_{0}
\end{array}\right) \\
& +\left(\begin{array}{c}
\tilde{q}_{1} \\
m
\end{array}\right)\left(\begin{array}{c}
\tilde{q}_{1}-1 \\
k-1
\end{array}\right)\left(\begin{array}{c}
m+q_{7}-1 \\
q_{7}
\end{array}\right)\left(\begin{array}{c}
k+q_{0} \\
q_{0}
\end{array}\right) .
\end{aligned}
$$

After rescaling to the new periodic orbit length $n^{\prime \prime}=n^{\prime}+q_{0}+q_{7}$ and summing the three contributions, one obtains

$$
P_{n^{\prime \prime}}\left(q_{0}, q_{2}, q_{5}, q_{7}\right)=\frac{n^{\prime \prime}}{\tilde{q}_{1}}\left(\begin{array}{c}
\tilde{q}_{1} \\
m
\end{array}\right)\left(\begin{array}{c}
\tilde{q}_{1} \\
k
\end{array}\right)\left(\begin{array}{c}
m+q_{7}-1 \\
q_{0}
\end{array}\right)\left(\begin{array}{c}
k+q_{0}-1 \\
q_{7}
\end{array}\right)
$$

with $\tilde{q}_{1}=\frac{1}{4}\left(n^{\prime \prime}-q_{0}+q_{2}+q_{5}-q_{7}\right)$ as in (A2). The final result (25) is obtained after noting that $m=q_{3}=q_{6}=\tilde{q}_{1}-q_{2}$ and $k=q_{1}=q_{4}=\tilde{q}_{1}-q_{5}$. Special care has to be taken in the case $q_{0}$ or $q_{7}=0$.

\section{The case $N=6$ :}

The periodic orbit length degeneracy function for $N=6$ can be derived by ideas similar to those outlined for $N=4$; I will sketch the main steps here and leave the details to the reader.

An edge symbol code is defined starting from the vertex symbol code used in Fig. 5 to be

$$
\begin{aligned}
& 00 \rightarrow 0_{e} ; \quad 01 \rightarrow 1_{e} \quad 12 \rightarrow 2_{e} ; \quad 13 \rightarrow 3_{e} \\
& 24 \rightarrow 4_{e} ; \quad 25 \rightarrow 5_{e} \quad 30 \rightarrow 6_{e} ; \quad 31 \rightarrow 7_{e} \\
& 42 \rightarrow 8_{e} ; \quad 43 \rightarrow 9_{e} \quad 54 \rightarrow 10_{e} ; \quad 55 \rightarrow 11_{e}
\end{aligned}
$$

A suitable set of independent edge staying rates is $q_{0_{e}}, q_{3_{e}}, q_{4_{e}}, q_{7_{e}}, q_{8_{e}}, q_{11_{e}}$ and I will drop the subscript $e$ from now on. The other edge staying rates of orbits of period $n$ are then given by

$$
\begin{aligned}
q_{1}=q_{6} & =\frac{1}{6}\left(n-q_{0}+3 q_{3}+q_{4}-5 q_{7}-3 q_{8}-q_{11}\right) \\
q_{2} & =\frac{1}{6}\left(n-q_{0}-3 q_{3}+q_{4}+q_{7}-3 q_{8}-q_{11}\right) \\
q_{5}=q_{10} & =\frac{1}{6}\left(n-q_{0}-3 q_{3}-5 q_{4}+q_{7}+3 q_{8}-q_{11}\right) ;
\end{aligned}
$$

the vertex rates are

$$
\tilde{q}_{0}=q_{0}+q_{1} ; \quad \tilde{q}_{1}=\tilde{q}_{3}=q_{2}+q_{3} ; \quad \tilde{q}_{2}=\tilde{q}_{4}=q_{4}+q_{5} ; \quad \tilde{q}_{5}=q_{10}+q_{11} .
$$

A suitable starting point for the periodic orbit length degeneracy function is the periodic orbit '2 49 7' (in edge code) or ' 1243 ' in vertex code, see Fig. 5. One obtains

$$
P_{n}\left(0,0, q_{4}=\frac{n}{4}, q_{7}=\frac{n}{4}, 0,0\right)=4 \quad \text { for } \quad n \bmod 4=0 .
$$


A symbol '7' can be followed by a loop '3 7' (with repetition), a symbol '4' may be followed by a loop '8 4' (with repetitions). Inserting $k$ loops ' 37 ' and $m$ loops ' 84 ' into a sequence '2 $497 \ldots$ ' of length $n-2 k-2 m$ yields

$$
\begin{aligned}
& P_{n}\left(0, k, \frac{1}{4}(n-2 k+2 m), \frac{1}{4}(n+2 k-2 m), l, 0\right)= \\
& 2\left(\begin{array}{c}
\frac{1}{4}(n+2 k-2 m)-1 \\
k
\end{array}\right)\left(\begin{array}{c}
\frac{1}{4}(n-2 k-2 m)-1 \\
m
\end{array}\right)+\left(\begin{array}{c}
\frac{1}{4}(n+2 k-2 m) \\
k
\end{array}\right)\left(\begin{array}{c}
\frac{1}{4}(n-2 k-2 m)-1 \\
m
\end{array}\right) \\
& +\left(\begin{array}{c}
\frac{1}{4}(n+2 k-2 m)-1 \\
k
\end{array}\right)\left(\begin{array}{c}
\frac{1}{4}(n-2 k-2 m) \\
m
\end{array}\right)+\left(\begin{array}{c}
\frac{1}{4}(n+2 k-2 m)-1 \\
k-1
\end{array}\right)\left(\begin{array}{c}
\frac{1}{4}(n-2 k-2 m)-1 \\
m
\end{array}\right) \\
& +\left(\begin{array}{c}
\frac{1}{4}(n+2 k-2 m)-1 \\
k
\end{array}\right)\left(\begin{array}{c}
\frac{1}{4}(n-2 k-2 m)-1 \\
m-1
\end{array}\right),
\end{aligned}
$$

and the different terms in the sum correspond to a first symbol in the periodic orbit code being either '2' or '9', '7', '4', '3' or '8', respectively. Next, one notes that every symbol '7' or '4' can be replaced by the sequence ' 6 1' or '5 10', respectively. I omit the somewhat lengthy combinatorial expressions here. The full periodic orbit length degeneracy function is finally obtained after inserting symbols ' 0 ' or '11' into the sequences '6 1' or '5 10', respectively, and summing over the various binomial coefficients. 